\title{
Evaluation of different hydrocortisone treatment strategies in transsphenoidal pituitary surgery
}

\author{
Ola Fridman-Bengtsson ${ }^{1,2}$ (1) C Charlotte Höybye ${ }^{3}$. Laura Porthén ${ }^{4}$ Pär Stjärne ${ }^{1,2} \cdot$ Anna-Lena Hulting $^{5}$. \\ Ola Sunnergren ${ }^{6}$
}

Received: 8 January 2019 / Accepted: 20 March 2019 / Published online: 7 May 2019

(C) The Author(s) 2019

\begin{abstract}
Background Hydrocortisone treatment in transsphenoidal pituitary surgery has been debated. Although several publications advocate restrictive treatment, centers around the world administer stress doses of hydrocortisone in patients with presumed intact cortisol production. Our aim with this analysis was to compare postoperative hypocortisolism in patients who received three different protocols of hydrocortisone therapy during and after surgery.

Method This was a retrospective observational study. Based on perioperative hydrocortisone dose given, patients were divided in three groups: high dose (HD), intermediate dose (ID), and low dose (LD). Postoperative evaluation of the pituitary function was performed using S-cortisol at day 4 and short Synacthen test (SST) at 6-8 weeks. Patients with ACTH-producing adenomas or preoperative hydrocortisone treatment were excluded.

Result There was no difference between the groups regarding failure rate of SST. The rate of failed SST (all groups) was 51/186 (27\%), 24/74 (32\%) in the HD group and 26/74 (35\%) and 11/38 (29\%) in the ID and LD groups respectively. There was no significant difference between the ID and LD groups regarding S-cortisol at postoperative day 4 regarding serum cortisol level below $200 \mathrm{nmol} / \mathrm{L}$. There was a significant but weak correlation, $r_{\mathrm{s}} 0.330(P<0.01)$ between S-cortisol day 4 and SST at $4-6$ weeks. Conclusions Peri and postoperative hydrocortisone treatment did not affect SST response 6-8 weeks postoperatively, whereas the rate of patients with S-cortisol below $200 \mathrm{nmol} / \mathrm{L}$ at postoperative day 4 did. LD hydrocortisone therapy seems to favor a better endogenous production in the early postoperative phase.
\end{abstract}

Keywords Hydrocortisone · Transsphenoidal · Pituitary surgery $\cdot$ Serum cortisol $\cdot$ Short synacthen test $\cdot$ Hypocortisolism

\section{Introduction}

The necessity of perioperative corticosteroid treatment during pituitary surgery has been debated over the years $[7,10,15,19,21,25,26,27]$. In the 1950s, Fraser et al.

This article is part of the Topical Collection on Pituitaries

Ola Fridman-Bengtsson

ola.fridman-bengtsson@sll.se

1 Department of Otorhinolaryngology, Karolinska University Hospital, Eugeniavägen 3, 171 76, Stockholm, Sweden

2 Department of Clinical Sciences, Intervention and Technology, Division of Otorhinolaryngology, Karolinska Institute, Stockholm, Sweden

3 Department of Molecular Medicine and Surgery, Patient Area Endocrinology and Nephrology, Inflammation and Infection Theme, Karolinska University Hospital, Stockholm, Sweden and Lewis et al. among others presented case reports with fatal outcome in patients with impaired hypothalamuspituitary-adrenal (HPA) axis undergoing surgery without cortisone treatment $[6,13]$. As a result of these reports, a strategy of giving stress doses of cortisone to all patients

4 Department of Medicine, Västmanland County Hospital, Västerås, Sweden

5 Department of Molecular Medicine and Surgery, Patient Area Endocrinology and Nephrology, Inflammation and Infection Theme, Karolinska University Hospital, Karolinska Institute, Stockholm, Sweden

6 Department of Otorhinolaryngology, Ryhov County Hospital, Futurum- the Academy for Health and Care, Jönköping, Sweden 
undergoing pituitary surgery was introduced $[4,8,15,20$, 23].

In 2002, Inder et al. published treatment guidelines on cortisone therapy during and after pituitary surgery [7]. These guidelines were based on retrospective studies and suggested restricted cortisone treatment to patients with intact or presumed intact function of the HPA axis. Since 2002, few prospective studies have been published supporting the safety of a more restrictive use of cortisone treatment in pituitary surgery $[11,26]$. Furthermore, one study even indicated that patients with impaired hypothalamic-pituitary-adrenal (HPA) axis managed without exogenous supply of cortisone during pituitary surgery [5].

Despite the 2002 guidelines, high doses of cortisone are still used in patients undergoing pituitary surgery in some centers around the world. One of the reasons may be difficulties in diagnosing and defining a suboptimal function of the HPA axis $[1,3,24]$. Other reasons may be local traditions - but also fear of inadequate pituitary function triggered by pituitary surgery.

At the Karolinska University Hospital, treatment schedules with high doses of hydrocortisone (i.e., a total of $970 \mathrm{mg}$ during the week of surgery) were until 2010 used in all patients undergoing pituitary surgery, irrespective of the function of the HPA axis. In 2010, the postoperative hydrocortisone treatment was reduced due to a high frequency of reported symptoms of hypercortisolism. Then, after evaluation of the reduced hydrocortisone doses in November 2016 a further reduction of the perioperative cortisol dose was introduced in line with current international trends.

The aim of this study was to describe and evaluate postoperative morning S-cortisol and cortisol reservoir after transsphenoidal endoscopic pituitary surgery in relation to different hydrocortisone treatment schedules.

\section{Methods and materials}

\section{Study design}

This was a retrospective observational study based on data from medical records of patients with non-ACTH producing adenomas nor preoperative hydrocortisone treatment, who underwent transsphenoidal endoscopic pituitary surgery at the Karolinska University Hospital. Based on the perioperative hydrocortisone dose used, patients were divided in three groups: high dose (HD), intermediate dose (ID), and low dose (LD) (Table 1). Patients in the HD group were included 2008 to 2009, patients in the ID group 2010 to 2012, and patients in the LD group 2016 to 2017.

Analyses of blood samples were performed at the Department of Clinical Chemistry, Karolinska University Hospital, using electrochemical luminescence, (Modular E,
Table 1 Hydrocortisone treatment in the different groups

\begin{tabular}{llll}
\hline & HD & ID & LD \\
\hline Day of surgery & $300 \mathrm{mg}^{*}$ & $300 \mathrm{mg}^{*}$ & $150 \mathrm{mg}^{*}$ \\
POD 1 & $200 \mathrm{mg}^{*}$ & $120 \mathrm{mg}^{* *}$ & $60 \mathrm{mg}^{* *}$ \\
POD 2 & $120 \mathrm{mg}^{* *}$ & $50 \mathrm{mg}^{* *}$ & $30 \mathrm{mg}^{* *}$ \\
POD 3 & $80 \mathrm{mg}^{* *}$ & $* * *$ & $* * *$ \\
POD 4 & $80 \mathrm{mg}^{* *}$ & & \\
POD 5 & $60 \mathrm{mg}^{* *}$ & & \\
POD 6 & $30 \mathrm{mg}^{* *}$ & & \\
\hline
\end{tabular}

$P O D 1$, first day after the day of surgery

*Intravenous hydrocortisone (Solucortef())

**Oral hydrocortisone

***Dosage according to the morning S-cortisol level.

Roche Diagnostics, Mannheim, Germany) who also supplied normative data. Due to a shift of reagents in 2016, S-cortisol values from HD and ID groups were recalculated in accordance with given instructions for comparison. As shown in Table 1, all patients operated during the period when the HD cortisol treatment schedule was used were prescribed cortisol for several weeks after surgery, i.e., until the short synacthen test (SST) at follow-up. S-cortisol was not analyzed in the HD group during the postoperative period. During the periods where ID and LD cortisol schedules were used, a morning S-cortisol was measured postoperatively at day 4 and used as an indicator for the need of continuous hydrocortisone treatment. An arbitrary level of $<200 \mathrm{nmol} / \mathrm{L}$ was routinely used as cutoff in assessing the risk of hypocortisolism. Day 4 was chosen, anticipating that surgical stress was over [2], and that the duration (day 1-3) of hydrocortisone treatment was too short to significantly hamper the HPA axis.

In all patients, postoperative evaluation of the pituitary function was performed after 6-8 weeks, including SST with measurements of S-cortisol before and $30 \mathrm{~min}$ after intravenous injection of $0.25 \mathrm{mg}$ Synacthen. A sufficient response was defined as a 30-min S-cortisol concentration above $450 \mathrm{nmol} / \mathrm{L}$ [12] .

\section{Statistical analysis}

Descriptive statistics were used for population and group characteristics. Independent $t$ test, chi-square, Kruskal-Wallis test, Spearman correlation, cross-tabulation, and ANOVA were used for group comparisons. All data were analyzed and graphically produced with SPSS statistical software version 24.0 (SPSS Inc., Chicago, IL). Statistical significance was set at $P<0.05$.

\section{Results}

A total of 186 patients, HD group $n=74$, ID group $n=74$, and LD group $n=38$, were included in the study (Fig. 1). General 
characteristics are presented in Table 2. There were no significant differences between the groups regarding age or gender. Regarding adenoma type, the groups were also comparable without significant disparity. Slightly fewer microadenomas were included in the LD group.

\section{Day 4 S-cortisol}

The results of S-cortisol at postoperative day 4 are presented in Table 2, Figs. 2 and 4. There was no statistically significant difference between the ID and LD groups regarding the number of patients with S-cortisol at postoperative day 4 with S-cortisol levels below $200 \mathrm{nmol} / \mathrm{L}$. In the ID group, $25 / 74(34 \%)$ had a S-cortisol below $200 \mathrm{nmol} / \mathrm{L}$ day 4 as compared with 11/38 (29\%) in the LD group.

Of the patients with S-cortisol below $200 \mathrm{nmol} / \mathrm{L}$ day 4, 14/ $25(56 \%)$ in the ID group failed SST, as compared with 6/11 $(55 \%)$ in the LD group. The distribution of day 4 S-cortisol and SST response are presented in Figs. 2 and 3 respectively and the diversity of measurements in Fig. 4.
Similar rates of failure at SST, in patients with S-cortisol above $200 \mathrm{nmol} / \mathrm{L}$, were observed in the ID group 12/49 $(24 \%)$ and in the LD group 5/27 (19\%).

\section{Short Synacthen test}

The results of SST at follow-up are presented by group in Figs. 1 and 4. There was no difference between the groups regarding failure rate of SST. The rate of failed SST (all groups) was 51/186 (27\%). Specifically, the failure rate was $24 / 74(32 \%)$ in the HD group and 26/74 (35\%) and 11/38 $(29 \%)$ in the ID and LD groups respectively. Neither gender nor age did significantly increase the risk of impaired response to SST in any of the groups at follow-up.

Patients who were treated with postoperative hydrocortisone (all groups) until follow-up failed SST in 47/113 (42\%). In the HD group 24/74 (32\%) failed SST, while 17/ $27(63 \%)$ and 6/12 (50\%) failed SST in the ID and LD groups respectively.

Patients not treated with postoperative hydrocortisone until follow-up (ID and LD groups only), failed SST in 14/73
Fig. 1 Flowchart of results of SST at follow-up

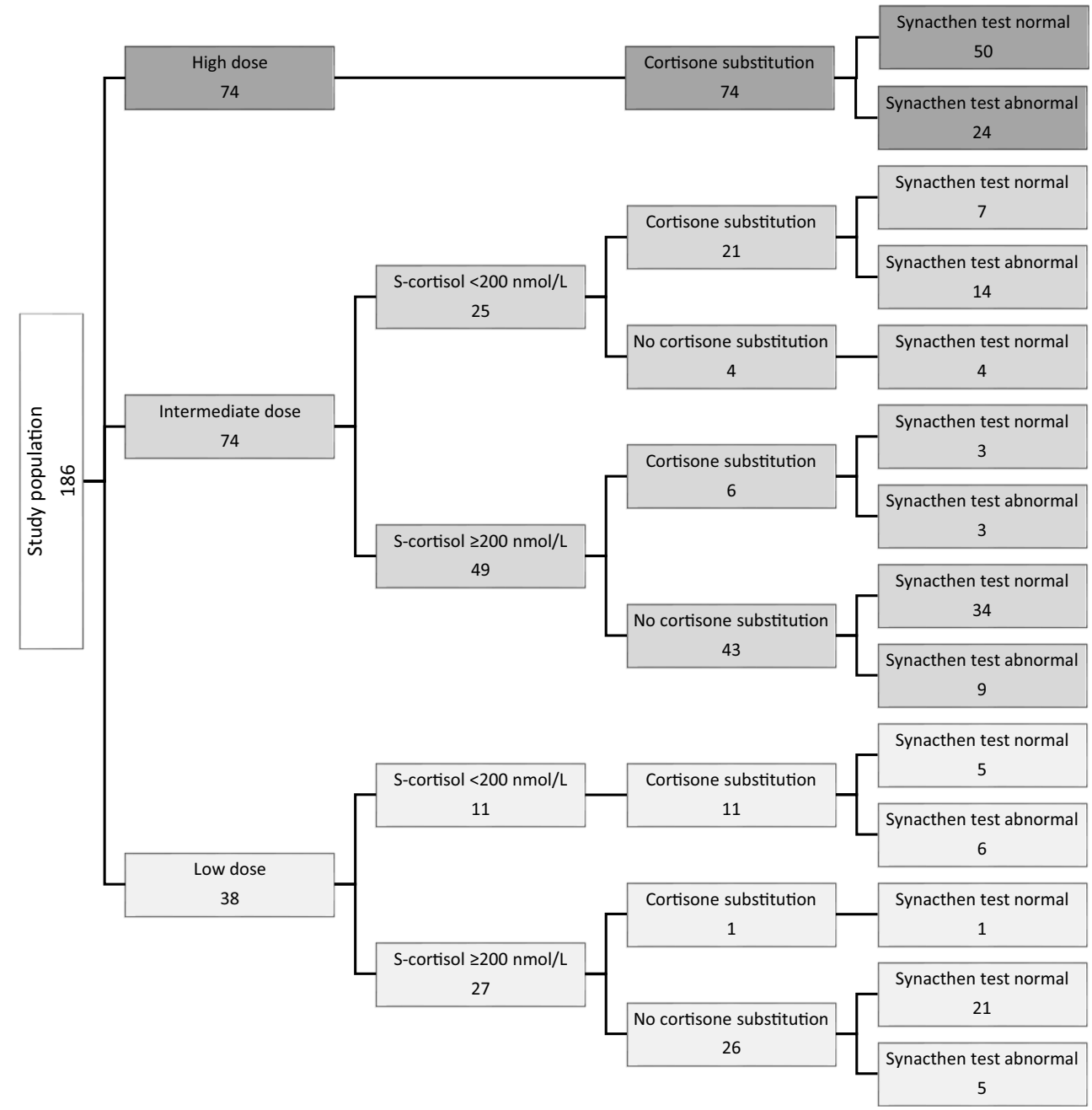


Table 2 General characteristics of the study population

\begin{tabular}{lllll}
\hline & HD & ID & LD & $P$ value \\
\hline$N$ & 74 & 74 & 38 & \\
Age (years, mean \pm SD) & $54.9 \pm 16$ & $51.6 \pm 16$ & $54.5 \pm 14$ & 0.397 \\
Females $n(\%)$ & $37(50)$ & $39(53)$ & $17(45)$ & 0.730 \\
Adenoma type & & & & \\
Inactive $(n)$ & 44 & 46 & 22 & 12 \\
GH $(n)$ & 8 & 20 & 0 & 0.252 \\
PRL $(n)$ & 3 & 3 & 1 & 0.047 \\
TSH $(n)$ & 0 & 0 & 3 & \\
Cysts $(n)$ & 8 & 5 & $2 / 36$ & \\
Micro/macroadenoma & $12 / 62$ & $9 / 65$ & 12 & \\
S-cortisol day 4 (nmol/L, mean \pm SD) & - & $275 \pm 164$ & $349 \pm 217$ & \\
Cortisone substitution from day 4 to follow-up $(n)$ & 74 & 27 & $11 / 38(29)$ & 0.058 \\
Inadequate Synacthen response at follow-up $n(\%)$ & $24 / 74(32)$ & $26 / 74(35)$ & &
\end{tabular}

(19\%). In the ID group 9/47 (19\%) failed SST, while 5/26 (19\%) failed SST in the LD group.

\section{Relation between short- and long-term evaluation of pituitary function}

There was a significant but weak correlation, $r_{\mathrm{s}} 0.330$ $(P<0.01)$ between S-cortisol day 4 and SST at 4-6 weeks. The positive predictive value of S-cortisol cutoff level at 200 at day 4 in relation to SST at 4-6 weeks follow-up was $77.6 \%$ while the negative predictive value was $55.6 \%$.

\section{Discussion}

In this retrospective study, we found no differences in the number of patients with insufficient response to SST between three groups with markedly different doses of perioperative hydrocortisone treatment after transsphenoidal pituitary surgery. A trend towards a lower rate of patients with S-cortisol less than $200 \mathrm{nmol} / \mathrm{L}$ at postoperative day 4 was seen in the group, who received the lowest dose (LD group). The rate of patients with S-cortisol postoperative day 4 above $200 \mathrm{nmol} / \mathrm{L}$ was similar in the two groups (ID and LD groups) where this variable was measured. Our study shows that $\mathrm{S}$-cortisol at day
Fig. 2 S-cortisol at postoperative day 4, ID and LD groups

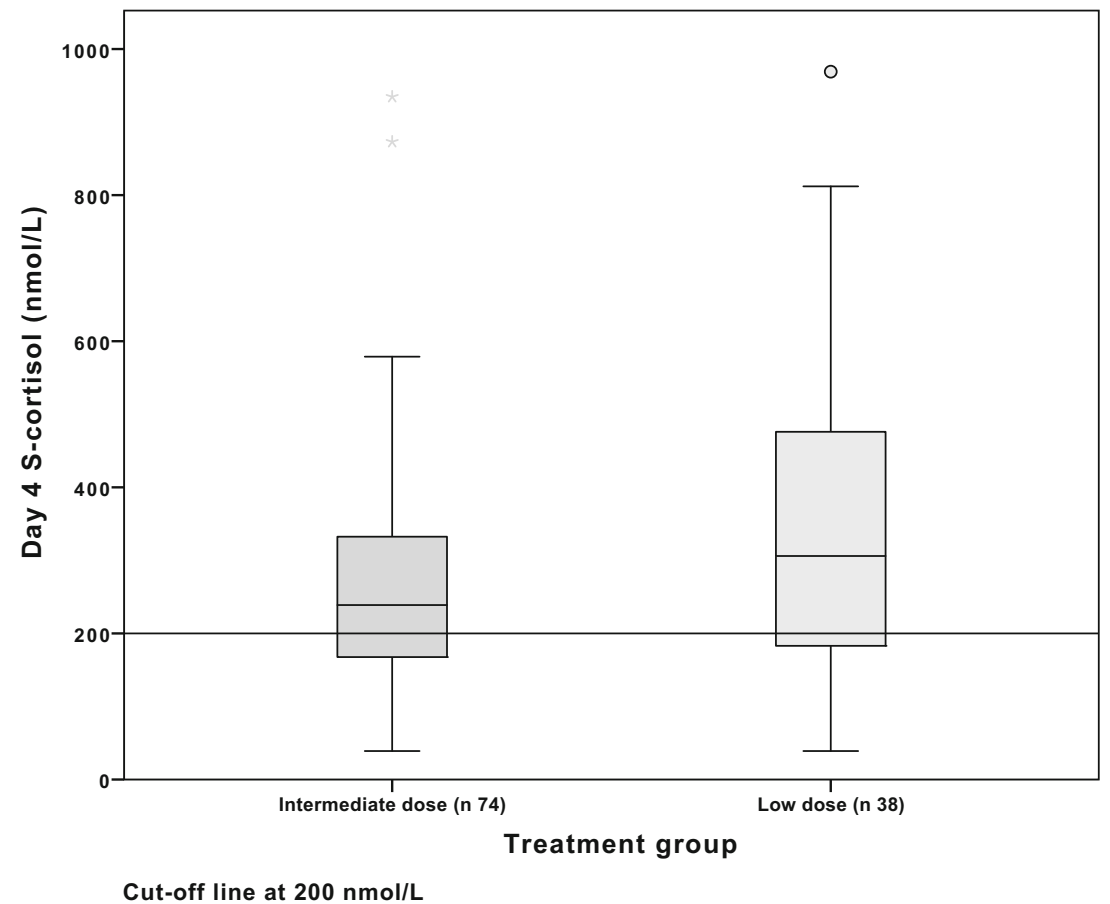


Fig. 3 Synacthen response at follow-up by group

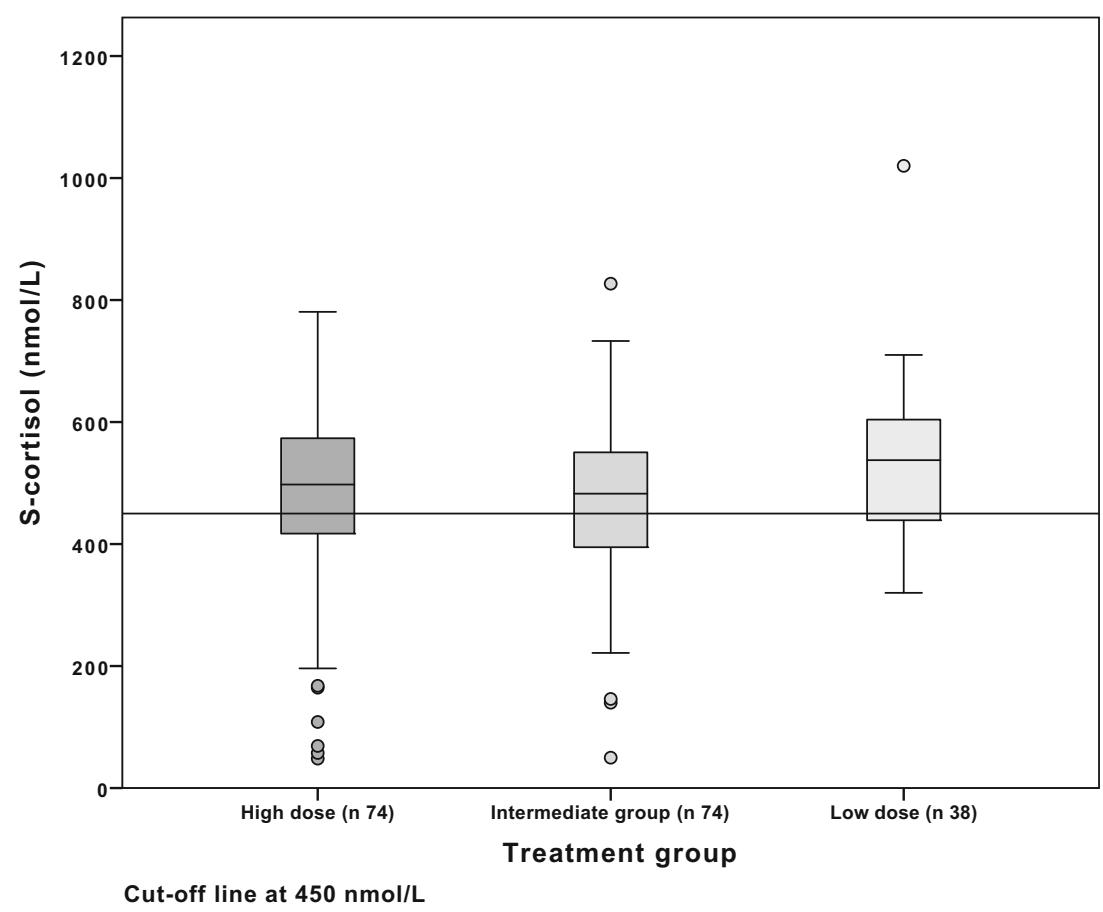

4 above $200 \mathrm{nmol} / \mathrm{L}$ predicts a normal SST within $77.6 \%$ of the patients in comparison with previous material [17]. Insufficient response to SST at follow-up seemed not to be dependent of perioperative hydrocortisone dose, whereas the rate of patients with S-cortisol below $200 \mathrm{nmol} / \mathrm{L}$ did. These results may point towards an inhibitory effect on S-cortisol day 4 related to the dose of perioperative hydrocortisone, showing higher response when the lowest dose (LD) was given.

In our patients, the preoperative function of the HPA-axis was assessed per the patient's clinical status and the level of morning S-cortisol. The time from diagnostics to elective pituitary surgery is short in our hospital (in general a few weeks) and as all patients undergoing pituitary surgery received
Fig. 4 Scatterplot of day 4 serum cortisol and Synacthen response at follow-up

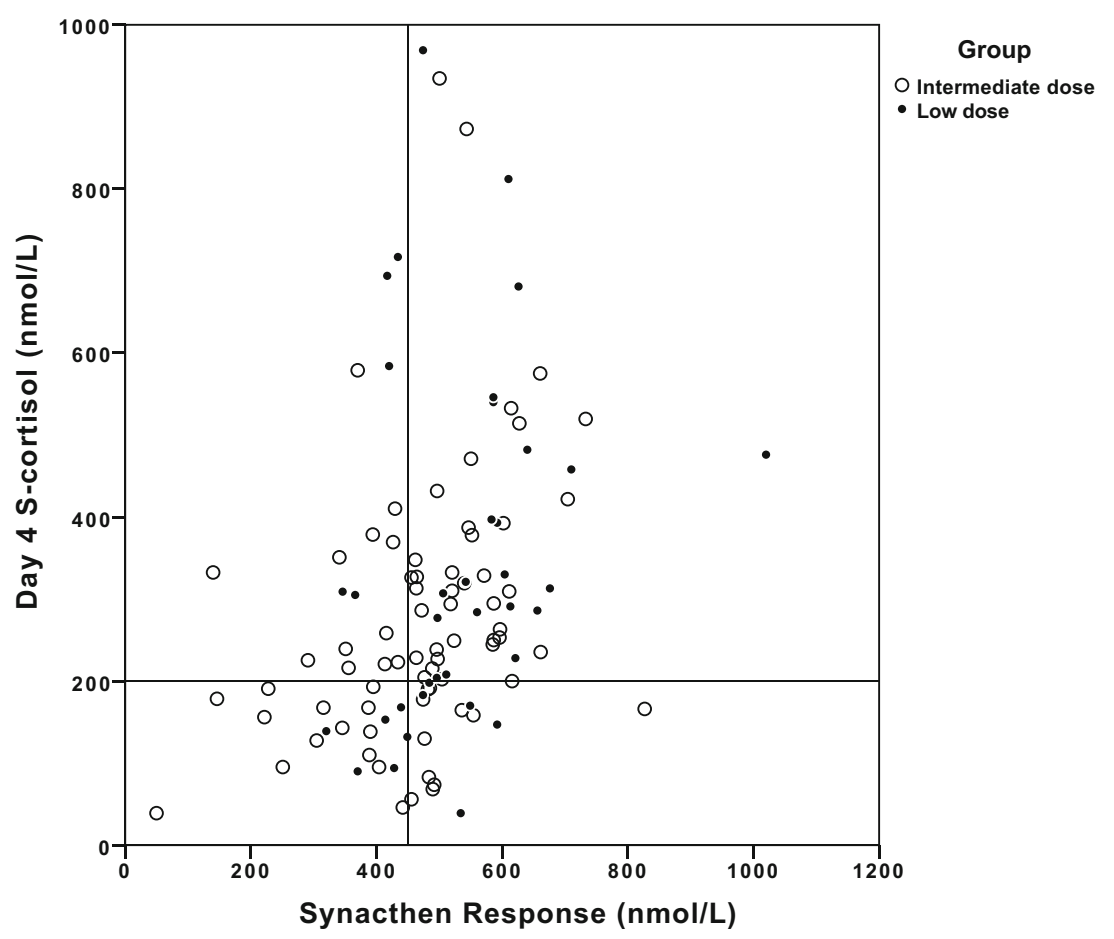

Cut-off lines at $200 \mathrm{nmol} / \mathrm{L}$ and $450 \mathrm{nmol} / \mathrm{L}$ respectively 
hydrocortisone treatment, although using different schedules, no further preoperative biochemical evaluation of the HPA-axis was performed. The size of the tumor and the presence of other pituitary deficiencies were other risk factors to perioperative hypocortisolism, which were considered. A single morning cortisol has been shown to be diagnostic of adrenal insufficiency if the level is below $100 \mathrm{nmol} / \mathrm{L}$ [12]. Our results indicate that $\mathrm{S}$-cortisol < $200 \mathrm{nmol} / \mathrm{L}$ seems to be an acceptable level to identify patients who need continuous hydrocortisone replacement in accordance with previous publications [9, 14, 16-18]. However, the diversity of S-cortisol at day 4 in our study indicates that, to predict long-term HPA-axis dysfunction at this point, the cutoff level must be significantly increased (Fig. 4). Doing so though, would imply less specificity to the test. We therefore argue that an arbitrary limit of morning cortisol must always be related to the patient's clinical status. Consequently, some of our patients with suspected symptoms of hypocortisolism continued with hydrocortisone after day 4 despite having levels $>200 \mathrm{nmol} / \mathrm{L}$. The reasons for this were in most cases, suspected or verified postoperative infections, and/or values close to cutoff level.

It is a challenge to balance the risk of hypocortisolism and the risk of overtreatment with hydrocortisone in patients undergoing pituitary surgery. During the period where the HD schedule was used, practically no patients were at risk of hypocortisolism. On the other hand, some patients were treated unnecessarily with hydrocortisone for weeks, and thereby at risk for unwanted side effects of hydrocortisone therapy. In contrast, the use of lower hydrocortisone doses and discontinuing this treatment after a few days might put some patients at risk of hypocortisolism. Low-dose schedules therefore imply the need for a close and thorough monitoring of the patients until the SST is performed. Discontinuation of hydrocortisone is also beneficial considering a simpler postoperative period without the need of hydrocortisone and decisions on changing the dose of it. Use of lower hydrocortisone doses and evaluation on day 4 has, in our experience, simplified the management of patients in the postoperative period after pituitary surgery.

It is a limitation that data was retrospectively collected from the medical records and not from a prospective, systematic controlled study.

In summary, we could not correlate any differences in longterm HPA-axis function in three different hydrocortisone treatment modalities. We found a weak but significant correlation between low day 4 S-cortisol and failed SST at follow-up, in line with the diversity of previous publications $[17,22]$. We observed no clinically significant impairment using lower hydrocortisone doses. Our results add to previous knowledge and recommendations, and conclude that an individualized regimen for hydrocortisone treatment is recommended in these patients.

\section{Compliance with ethical standards}

Conflict of interest The authors declare that they have no conflict of interest.

Ethical approval All procedures performed in studies involving human participants were in accordance with the ethical standards of the institutional and/or national research committee at the Karolinska Institute (2012/1689-31/4) and with the 1964 Helsinki declaration and its later amendments or comparable ethical standards.

Informed consent For this type of study, no formal consent is not required.

Open Access This article is distributed under the terms of the Creative Commons Attribution 4.0 International License (http:// creativecommons.org/licenses/by/4.0/), which permits unrestricted use, distribution, and reproduction in any medium, provided you give appropriate credit to the original author(s) and the source, provide a link to the Creative Commons license, and indicate if changes were made.

\section{References}

1. Agha A, Tomlinson JW, Clark PM, Holder G, Stewart PM (2006) The long-term predictive accuracy of the short synacthen (corticotropin) stimulation test for assessment of the hypothalamic-pituitary-adrenal axis. J Clin Endocrinol Metab 91: 43-47

2. Borg H, Siesjo P, Kahlon B, Fjalldal S, Erfurth EM ([2018) Perioperative serum cortisol levels in ACTH sufficient and ACTH deficient patients during transsphenoidal surgery of pituitary adenoma. Endocrine 62:83-89

3. Chatha KK, Middle JG, Kilpatrick ES (2010) National UK audit of the short synacthen test. Ann Clin Biochem 47:158-164

4. de Lange DW, Kars M (2008) Perioperative glucocorticosteroid supplementation is not supported by evidence. Eur J Int Med 19: 461-467

5. De Tommasi C, Goguen J, Cusimano MD (2012) Transphenoidal surgery without steroid replacement in patients with morning serum cortisol below 9 mug/dl (250 Nmol/l). Acta Neurochir 154:19031915

6. Fraser CG, Preuss FS, Bigford WD (1952) Adrenal atrophy and irreversible shock associated with cortisone therapy. J Am Med Assoc 149:1542-1543

7. Inder WJ, Hunt PJ (2002) Glucocorticoid replacement in pituitary surgery: guidelines for perioperative assessment and management. $J$ Clin Endocrinol Metab 87:2745-2750

8. Jabbour SA (2001) Steroids and the surgical patient. Med Clin North Am 85:1311-1317

9. Jayasena CN, Gadhvi KA, Gohel B, Martin NM, Mendoza N, Meeran K, Dhillo WS (2009) Day 5 morning serum cortisol predicts hypothalamic-pituitary-adrenal function after transsphenoidal surgery for pituitary tumors. Clin Chem 55:972-977

10. Joseph SP, Ho JT, Doogue MP, Burt MG (2012) Perioperative management of the hypothalamic-pituitary-adrenal axis in patients with pituitary adenomas: an Australasian survey. Intern Med J 42:11201124

11. Kristof RA, Wichers M, Haun D, Redel L, Klingmuller D, Schramm J (2008) Peri-operative glucocorticoid replacement therapy in transsphenoidal pituitary adenoma surgery: a prospective controlled study. Acta Neurochir 150:329-335 discussion 335 
12. Le Roux CW, Meeran K, Alaghband-Zadeh J (2002) Is a 0900-h serum cortisol useful prior to a short synacthen test in outpatient assessment? Ann Clin Biochem 39:148-150

13. Lewis L, Robinson RF, Yee J, Hacker LA, Eisen G (1953) Fatal adrenal cortical insufficiency precipitated by surgery during prolonged continuous cortisone treatment. Ann Intern Med 39: 116-126

14. Manuylova E, Calvi LM, Vates GE, Hastings C, Shafiq I (2015) Morning serum cortisol level after transsphenoidal surgery for pituitary adenoma predicts hypothalamic-pituitary-adrenal function despite intraoperative dexamethasone use. Endocrine practice : official journal of the American College of Endocrinology and the American Association of Clinical Endocrinologists 21:897-902

15. Marik PE, Varon J (2008) Requirement of perioperative stress doses of corticosteroids: a systematic review of the literature. Arch Surg(Chicago, Ill : 1960) 143:1222-1226

16. Marko NF, Gonugunta VA, Hamrahian AH, Usmani A, Mayberg MR, Weil RJ (2009) Use of morning serum cortisol level after transsphenoidal resection of pituitary adenoma to predict the need for long-term glucocorticoid supplementation. J Neurosurg 111: $540-544$

17. Marko NF, Hamrahian AH, Weil RJ (2010) Immediate postoperative cortisol levels accurately predict postoperative hypothalamicpituitary-adrenal axis function after transsphenoidal surgery for pituitary tumors. Pituitary 13:249-255

18. McLaughlin N, Cohan P, Barnett P, Eisenberg A, Chaloner C, Kelly DF (2013) Early morning cortisol levels as predictors of short-term and long-term adrenal function after endonasal transsphenoidal surgery for pituitary adenomas and Rathke's cleft cysts. World neurosurgery 80:569-575

19. Nemergut EC, Dumont AS, Barry UT, Laws ER (2005) Perioperative management of patients undergoing transsphenoidal pituitary surgery. Anesth Analg 101:1170-1181
20. Plumpton FS, Besser GM, Cole PV (1969) Corticosteroid treatment and surgery. 2. The management of steroid cover. Anaesthesia 24: $12-18$

21. Prete A, Corsello SM, Salvatori R (2017) Current best practice in the management of patients after pituitary surgery. Ther Adv Endocrinol Metab 8:33-48

22. Ramm-Pettersen J, Halvorsen H, Evang JA, Ronning P, Hol PK, Bollerslev J, Berg-Johnsen J, Helseth E (2015) Low immediate postoperative serum-cortisol nadir predicts the short-term, but not long-term, remission after pituitary surgery for Cushing's disease. BMC Endocr Disord 15:62

23. Regan J, Watson J (2013) Selective use of peri-operative steroids in pituitary tumor surgery: escape from dogma. Front Endocrinol 4:30

24. Reynolds RM, Stewart PM, Seckl JR, Padfield PL (2006) Assessing the HPA axis in patients with pituitary disease: a UK survey. Clin Endocrinol 64:82-85

25. Tohti M, Li J, Zhou Y, Hu Y, Yu Z, Ma C (2015) Is peri-operative steroid replacement therapy necessary for the pituitary adenomas treated with surgery? A systematic review and meta analysis. PLoS One 10:e119621

26. Wentworth JM, Gao N, Sumithran KP, Maartens NF, Kaye AH, Colman PG, Ebeling PR (2008) Prospective evaluation of a protocol for reduced glucocorticoid replacement in transsphenoidal pituitary adenomectomy: prophylactic glucocorticoid replacement is seldom necessary. Clin Endocrinol 68:29-35

27. Zada G, Woodmansee WW, Iuliano S, Laws ER (2010) Perioperative management of patients undergoing transsphenoidal pituitary surgery. Asian J Neurosurg 5:1-6

Publisher's note Springer Nature remains neutral with regard to jurisdictional claims in published maps and institutional affiliations. 